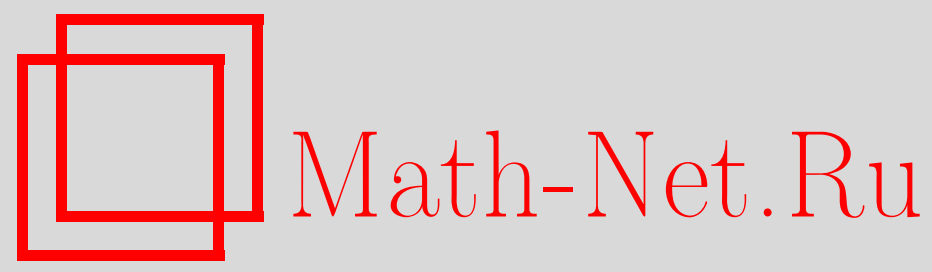

А. Ю. Колесов, Н. Х. Розов, Параметрическая буферность в сингулярно возмущенном телеграфном уравнении с маятниковой нелинейностью, Матем. заметки, 2001, том 69, выпуск 6, 866-875

DOI: https://doi.org/10.4213/mzm700

Использование Общероссийского математического портала Math-Net.Ru подразумевает, что вы прочитали и согласны с пользовательским соглашением http://www . mathnet.ru/rus/agreement

Параметры загрузки:

IP: 3.91 .87 .62

26 апреля 2023 г., 12:30:07

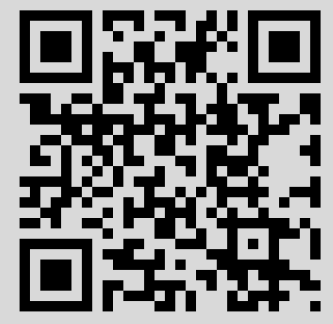




\title{
ПАРАМЕТРИЧЕСКАЯ БУФЕРНОСТЬ \\ В СИНГУЛЯРНО ВОЗМУЩЕННОМ ТЕЛЕГРАФНОМ УРАВНЕНИИ С МАЯТНИКОВОЙ НЕЛИНЕЙНОСТЬЮ
}

\author{
А. Ю. Колесов, Н. Х. Розов
}

Рассматривается краевая задача

$$
u_{t t}+\varepsilon u_{t}+(1+\varepsilon \alpha \cos 2 \tau) \sin u=\varepsilon \sigma^{2} u_{x x},\left.\quad u_{x}\right|_{x=0}=\left.u_{x}\right|_{x=\pi}=0,
$$

где $0<\varepsilon \ll 1, \tau=(1+\varepsilon \delta) t, \alpha, \sigma>0$, а знак $\delta$ произволен. Доказано существование (при подходящем выборе параметров внешнего воздействия $\alpha, \delta$ и при надлежащем уменьшении $\sigma$ ) любого фиксированного числа экспоненциально устойчивых $2 \pi$-периодических по $\tau$ решений.

Библиография: 14 названий.

Основы теории нелинейного параметрического резонанса для обыкновенных дифференциальных уравнений были заложены в монографии [1], а ее результаты распространены на некоторые классы волновых уравнений в [2]. Изучение параметрического резонанса в сингулярно возмущенных системах начато по инициативе Ю. С. Колесова в [3] для параболических систем типа реакция-диффузия с малой диффузией. В [4]-[7] проводился анализ параметрических колебаний в сингулярно возмущенных телеграфных уравнениях.

Как отмечено Колесовым [6], мотивацией для таких исследований служит то обстоятельство, что здесь мы оказываемся "в окрестности наиболее глубокой особенности". А это, в свою очередь, может позволить в полном объеме выявить все динамические эффекты, порождаемые параметрическим внешним воздействием.

Плодотворность такой точки зрения иллюстрируют результаты [8], где показано, что в параболических и гиперболических системах с малыми коэффициентами диффузии реализуется феномен параметрической буферности. Суть этого явления состоит в том, что при подходящем выборе параметров внешнего воздействия и при надлежашем уменьшении диффузии можно гарантировать существование в указанных системах любого фиксированного числа устойчивых периодических по времени решений.

Однако в [8] остался без рассмотрения важньй частный случай - телеграфное уравнение с маятниковой нелинейностью (так в теории колебаний принято называть нелинейную зависимость типа "sin $u$ " от искомой функции $u$ ). Отметим, что сингулярно возмущенное телеграфное уравнение впервые изучалось в [7], но явление параметрической буферности установлено там не было.

Работа выполнена при финансовой поддержке Российского фонда фундаментальных исследований, грант № 99-01-00157. 
Цель настоящей статьи - восполнить данный пробел.

1. Постановка задачи и описание результатов. На отрезке $0 \leqslant x \leqslant \pi$ рассмотрим краевую задачу

$$
u_{t t}+\varepsilon u_{t}+(1+\varepsilon \alpha \cos 2 \tau) \sin u=\varepsilon \sigma^{2} u_{x x},\left.\quad u_{x}\right|_{x=0}=\left.u_{x}\right|_{x=\pi}=0,
$$

где $0<\varepsilon \ll 1, \tau=(1+\varepsilon \delta) t$, параметры $\alpha, \sigma>0$ и параметр $\delta$, знак которого произволен, имеют порядок единицы.

Будем интересоваться вопросами существования и устойчивости периодических по $t$ решений краевой задачи $(1)$, бифурцируюших из нуля при $\varepsilon>0$. При этом устойчивость понимается в метрике фазового пространства (пространства начальных условий $\left.\left(u, u_{t}\right)\right) \stackrel{\circ}{W}_{2}^{2}(0, \pi) \times W_{2}^{1}(0, \pi)$, где $\stackrel{\circ}{W}_{2}^{2}, W_{2}^{1}$ - соболевские пространства функций, причем функции, входяшие в первое из них, удовлетворяют граничньм условиям из (1). Понятие фазового пространства позволяет практически без изменений переносить на различные классы операторных дифференциальных уравнений основныеположения теории нелинейных колебаний.

Для решения поставленной проблемы воспользуемся методом квазинормальных форм Колесова. Следуя методике работ [6], [7], подставим в $(1)$ ряд по нечетным степеням $\sqrt{\varepsilon}$ :

$$
u=\varepsilon^{1 / 2} u_{0}(s, \tau, x)+\varepsilon^{3 / 2} u_{1}(s, \tau, x)+\cdots,
$$

где

$$
s=\varepsilon t, \quad u_{0}=\xi(s, x) \exp (i \tau)+\bar{\xi}(s, x) \exp (-i \tau),
$$

все функции периодичны по $\tau$ с периодом $2 \pi$, а $\xi$ - неизвестная (подлежащая определению) комплексная “амплитуда". Приравнивая затем коэффициенты при $\varepsilon^{3 / 2}$, для нахождения $u_{1}$ приходим к обыкновенному линейному неоднородному дифференциальному уравнению, в котором зависяшая от $s, x$ как от параметров неоднородность - сумма первых и третьих гармоник по $\tau$. Поэтому для того чтобы его решение $u_{1}$ принадлежало нужному классу, в неоднородности следует приравнять к нулю коэффициенты при $\exp ( \pm i \tau)$. На этом пути для определения $\xi$ получаем краевую задачу

$$
\xi_{s}=-\frac{i}{2} \sigma^{2} \xi_{x x}-\left(\frac{1}{2}+i \delta\right) \xi+\frac{i \alpha}{4} \bar{\xi}-\frac{i}{4} \xi|\xi|^{2},\left.\quad \xi_{x}\right|_{x=0}=\left.\xi_{x}\right|_{x=\pi}=0,
$$

которую согласно терминологии [6] и [7] будем называть квазинормальной формой исходной задачи (1).

Справедливо следующее утверждение (см. [6], [7]).

ТЕОремА 1. Пусть квазинормальная форма (4) имеет әкспоненииально устойчивое или дихотомичное (в метрике $\dot{\circ}_{2}^{2}(0, \pi)$ ) состояние равновесия $\xi=\xi_{0}(x)$. Тогда при всех достаточно малых $\varepsilon>0$ в исходной краевой задаче (1) ему от-

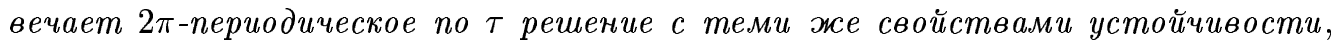
асимптотика которого с точностью до $\varepsilon^{3 / 2}$ получается из (2), (3).

Сформулированная теорема сводит вопрос о количестве и устойчивости $2 \pi$-периодических по $\tau$ решений краевой задачи (1) к выявлению количества и устойчивости состояний равновесия у ее квазинормальной формы (4). Обозначим через $\Omega_{n}$ совокупность параметров $(\sigma, \alpha, \delta)$, при которых (4) имеет не менее $n$ различных экспоненциально устойчивых состояний равновесия.

Наш основной результат - следующее утверждение. 
ТЕОРемА 2 (о параметрической буферности). Множество $\Omega_{n}$ непусто при любом натуральном $n$.

2. Существование высокомодовых состояний равновесия. При доказательстве теоремы 2 будем считать, что в (4) параметры имеют вид

$$
\alpha=2+4 \mu, \quad \frac{\sigma^{2}}{2}=\mu \beta^{2}, \quad \delta>0,
$$

где $0<\mu \ll 1, \beta=$ const $>0$. Полагая, далее, $w_{1}=\operatorname{Re} \xi, w_{2}=\operatorname{Im} \xi, w=\operatorname{colon}\left(w_{1}, w_{2}\right)$, перепишем при условиях (5) задачу (4) в вешественной форме:

$$
w_{s}=\mu \beta^{2} D w_{x x}+A_{0} w+\mu A_{1} w+\frac{1}{4}(w, w) D w,\left.\quad w_{x}\right|_{x=0}=\left.w_{x}\right|_{x=\pi}=0
$$

где

$$
D=\left(\begin{array}{cc}
0 & 1 \\
-1 & 0
\end{array}\right), \quad A_{0}=\left(\begin{array}{cc}
-\frac{1}{2} & \frac{1}{2}+\delta \\
\frac{1}{2}-\delta & -\frac{1}{2}
\end{array}\right), \quad A_{1}=\left(\begin{array}{ll}
0 & 1 \\
1 & 0
\end{array}\right)
$$

Поясним смысл ограничений (5). С этой целью отбросим в (6) нелинейность и применим к получившейся линейной краевой задаче метод Фурье по системе функций $\cos n x$, $n=0,1, \ldots$ В итоге убедимся, что за устойчивость ее нулевого состояния равновесия отвечает расположение спектра семейства матриц

$$
A_{0}+\mu A_{1}-\mu \beta^{2} n^{2} D, \quad n=0,1, \ldots
$$

Далее, введем в рассмотрение квадратичньй многочлен

$$
P_{\mu}(z)=(z-\delta)^{2}-\mu-\mu^{2}
$$

Несложный анализ показывает, что каждая матрица семейства (7) является гурвицевой в случае, когда $P_{\mu}\left(\mu \beta^{2} n^{2}\right)>0$, или имеет одно вещественное положительное собственное число в случае, когда $P_{\mu}\left(\mu \beta^{2} n^{2}\right)<0$. Таким образом, в задаче (6) возбуждены только моды $\cos n x$ с асимптотически высокими номерами $n$, удовлетворяюшими неравенствам

$$
z_{-}(\mu)<\mu \beta^{2} n^{2}<z_{+}(\mu)
$$

где $z_{ \pm}(\mu)=\delta \pm \sqrt{\mu+\mu^{2}}-$ корни многочлена (8) (их положительность вытекает из (5)). Отметим еще, что хотя количество номеров $n$, удовлетворяющих оценкам (9), асимптотически конечно (остается ограниченньм при $\mu \rightarrow 0$ ), оно может быть сделано сколь угодно большим за счет уменьшения параметра $\beta$. И наконец, характеристические показатели (положительные собственные числа матриц (7)) на этих модах малы, а точнее говоря, имеют порядок $\mu$. Тем самым, проясняется роль ограничений (5): они делают возможным применение к задаче (6) локальных бифуркационных методов.

Итак, проблема доказательства теоремы 2 сводится к вопросу о существовании и устойчивости состояний равновесия задачи (6), бифурцируюших из нуля при $\mu>0$ на модах с номерами (9). Для нахождения указанных состояний равновесия воспользуемся принципом подобия (см. [8], [9]).

Этот принцип включает в себя три этапа. 
Этап 1. Вьполним в (6) замену переменной $n x \rightarrow x$, где $n$ - достаточно большое натуральное число. В результате придем к задаче

$$
w_{s}=\left(\delta+\sqrt{\mu} \gamma_{n}(\mu)\right) D w_{x x}+A_{0} w+\mu A_{1} w+\frac{1}{4}(w, w) D w,\left.\quad w_{x}\right|_{x=0}=\left.w_{x}\right|_{x=n \pi}=0
$$

где $\gamma_{n}(\mu)=\left(\mu \beta^{2} n^{2}-\delta\right) / \sqrt{\mu}$ будем считать величиной порядка единишы.

Этап 2. Введем в рассмотрение вспомогательную задачу

$$
w_{s}=(\delta+\sqrt{\mu} \gamma) D w_{x x}+A_{0} w+\mu A_{1} w+\frac{1}{4}(w, w) D w,\left.\quad w_{x}\right|_{x=0}=\left.w_{x}\right|_{x=\pi}=0
$$

получающуюся из (10) заменой дискретного параметра $\gamma_{n}$ на непрерывный и переходом от отрезка $0 \leqslant x \leqslant n \pi$ на прежний отрезок $0 \leqslant x \leqslant \pi$. Предположим, далее, что на некотором промежутке

$$
\gamma_{1} \leqslant \gamma \leqslant \gamma_{2}
$$

изменения параметра $\gamma$ эта задача имеет нетривиально зависяшее от $x$ состояние равновесия

$$
w=w_{0}(x, \gamma, \mu)
$$

Продолжим это решение по $x$ на отрезок $-\pi \leqslant x \leqslant 0$ четным образом, а затем на всю ось $x$ по периодичности с периодом $2 \pi$. Тогда, как легко видеть, оно будет удовлетворять граничным условиям из (10) при любом натуральном $n$, а при $\gamma=\gamma_{n}(\mu) \in\left[\gamma_{1}, \gamma_{2}\right]$ всей задаче (10).

Этап 3. Возвращаясь к исходной задаче (6), заключаем, что при сделанных предположениях она имеет высокомодовые состояния равновесия

$$
w_{n}^{1}(x, \mu)=w_{0}\left(n x, \gamma_{n}(\mu), \mu\right), \quad w_{n}^{2}(x, \mu)=w_{0}\left(\pi-n x, \gamma_{n}(\mu), \mu\right)
$$

с номерами $n$, удовлетворяюшими неравенствам

$$
\frac{1}{\beta \sqrt{\mu}} \sqrt{\delta+\gamma_{1} \sqrt{\mu}} \leqslant n \leqslant \frac{1}{\beta \sqrt{\mu}} \sqrt{\delta+\gamma_{2} \sqrt{\mu}} .
$$

Сформулированньй принцип сводит исследуемую проблему к нахождению пространственно неоднородных состояний равновесия вспомогательной задачи (11), регулярно зависящей от всех своих параметров. Заметим, далее, что поскольку при $\mu=0$ линеаризованная в нуле задача (11) имеет простой нулевой характеристический показатель, которому отвечает решение $\Phi$ локе $w=a \cos x, a=\operatorname{colon}(1,1)$, а все остальные ее характеристические показатели имеют отрицательные действительные части, то речь может идти только о ее состояниях равновесия, бифурцирующих из нуля при $\mu>0$ на моде $\cos x$.

Для построения асимптотики упомянутых состояний равновесия задачи (11) воспользуемся аналогом одночастотного метода (см. [1]), т.е. подставим в (11) ряд по нечетным степеням $\mu^{1 / 4}$ :

$$
w=\mu^{1 / 4} w_{0}(x)+\mu^{3 / 4} w_{1}(x)+\mu^{5 / 4} w_{2}(x)+\cdots,
$$

где

$$
w_{0}(x)=\eta_{0} a \cos x
$$


а $\eta_{0}$ - неизвестная (подлежащая определению) вещественная постоянная. Приравнивая затем коэффишиенты при $\mu^{3 / 4}$ и $\mu^{5 / 4}$, для нахождения $w_{1}$ и $w_{2}$ получим краевые задачи (штрих - дифференцирование по $x$ ):

$$
\begin{gathered}
\delta D w_{1}^{\prime \prime}+A_{0} w_{1}+\gamma D w_{0}^{\prime \prime}+\frac{1}{4}\left(w_{0}, w_{0}\right) D w_{0}=0,\left.\quad w_{0}^{\prime}\right|_{x=0}=\left.w_{0}^{\prime}\right|_{x=\pi}=0 ; \\
\delta D w_{2}^{\prime \prime}+A_{0} w_{2}+\gamma D w_{1}^{\prime \prime}+A_{1} w_{0}+\frac{1}{2}\left(w_{0}, w_{1}\right) D w_{0}+\frac{1}{4}\left(w_{0}, w_{0}\right) D w_{1}=0 \\
\left.w_{2}^{\prime}\right|_{x=0}=\left.w_{2}^{\prime}\right|_{x=\pi}=0 .
\end{gathered}
$$

Анализ задачи (18) показывает, что в силу равенства $(D a, a)=0$ она оказывается разрешимой и ее решение имеет вид

$$
w_{1}=\eta_{1} a \cos x+a_{1}\left(\gamma \eta_{0}-\frac{3}{8} \eta_{0}^{3}\right) \cos x+\frac{\eta_{0}^{3}}{64 \delta} a \cos 3 x
$$

где $a_{1}=\operatorname{colon}(-1,1)$, а $\eta_{1}$ - произвольная вешественная постоянная. Подставляя, далее, (17) и (20) в (19), приходим к вьводу, что условие разрешимости задачи (19) задается равенством

$$
\Phi\left(\eta_{0}\right) \equiv 2\left(1-\gamma^{2}\right) \eta_{0}+\frac{3}{2} \gamma \eta_{0}^{3}-\frac{9}{32} \eta_{0}^{5}=0 .
$$

Предположим, что фигурируюший в (12) параметр $\gamma$ удовлетворяет условию $\gamma>-1$. Тогда, как легко видеть, уравнение (21) имеет решение

$$
\eta_{0}(\gamma)=\sqrt{\frac{8(\gamma+1)}{3}}
$$

для которого вьполняется неравенство

$$
\Phi^{\prime}\left(\eta_{0}\right)<0
$$

Подставляя его в (17) и (20), полностью определим первьй член ряда (16) и частично - второй. Что же касается фигурирующей в (20) произвольной постоянной $\eta_{1}$, то она определяется из условия разрешимости аналогичной (19) задачи для $w_{3}$, причем, как это обычно бывает в теории ветвления, для нее получается уже линейное неоднородное уравнение вида $\Phi^{\prime}\left(\eta_{0}\right) \eta_{1}=\varphi_{1}$. А так как в дальнейшем явная формула для $\eta_{1}$ не потребуется, то соответствуюшие вычисления опустим.

Лемма 1. По любым фиксированным $-1<\gamma_{1}<\gamma_{2}$ можнно указать достаточно малое $\mu_{0}=\mu_{0}\left(\gamma_{1}, \gamma_{2}\right)>0$ такое, что при всех $0<\mu \leqslant \mu_{0}$ и при значениях $\gamma$ из отрезка (12) краевая задача (11) имеет два әкспоненциально устойчивых состояния равновесия

$$
w_{1}(x, \gamma, \mu)=w_{0}(x, \gamma, \mu), \quad w_{2}(x, \gamma, \mu)=w_{0}(\pi-x, \gamma, \mu)
$$

где функиия $w_{0}(x, \gamma, \mu)$ допускает (в метрике $\stackrel{\circ}{2}_{2}^{2}\left([0, \pi] ; \mathbb{R}^{2}\right)$ ) асимптотику $(16)$, (17), (22).

Сформулированная лемма представляет собой один из вариантов классической бифуркационной теоремы Тьюринга-Пирожина [10]. Однако в отличие от классической 
ситуации, разложение в (16) идет не по степеням корня квадратного из надкритичности $\mu$, а по степеням $\mu^{1 / 4}$, что связано со спецификой фигурирующей в $(6)$ нелинейности. Именно по этой причине в случае задачи (1) теорема 2 не вытекает непосредственно из результатов [8], хотя применяемая нами общая схема исследования здесь, естественно, та же, что и в [8].

Для доказательства леммы 1 заметим, что при всех $0<\mu \ll 1$ задача (11) в окрестности нулевого состояния равновесия имеет экспоненциально орбитально устойчивое одномерное интегральное многообразие [11] $w=\eta a \cos x+H(\eta, \mu)$, уравнение на котором после нормировок $\eta / \mu^{1 / 4} \rightarrow \eta, \mu s \rightarrow s$ записывается в виде

$$
2 \frac{d \eta}{d s}=\Phi(\eta)+\sqrt{\mu} \Omega(\eta, \mu) .
$$

Здесь функция $\Phi$ определена в (21), а достаточно гладкие по совокупности переменных $\eta, \sqrt{\mu}$ функции $\Omega$ и $H$ со значениями в $\mathbb{R}$ и $\dot{W}_{2}^{2}\left([0, \pi] ; \mathbb{R}^{2}\right)$ соответственно таковы, что $H(0, \mu) \equiv 0, H_{\eta}^{\prime}(0,0)=0, \Omega(0, \mu) \equiv 0$. Таким образом, доказательство леммы сводится к исследованию состояний равновесия скалярного уравнения (25). Остается добавить, что последнее имеет состояние равновесия, асимптотически близкое к (22), а его устойчивость вытекает из неравенства (23).

Установленная лемма и описанный выше принцип подобия приводят к выводу, что при всех $0<\mu \ll 1$ исходная краевая задача (6) имеет высокомодовые состояния равновесия $(14),(15)$, где в качестве решения (13) фигурирует состояние равновесия $w_{0}(x, \gamma, \mu)$ из (24).

3. Исследование устойчивости высокомодовых решений. Линеаризуем задачу (6) на произвольном состоянии равновесия (14) с номером $n$ и вьполним замену $y=n x$. В результате получим спектральную задачу

$$
\begin{gathered}
(\delta+\sqrt{\mu} \gamma) D h^{\prime \prime}+\left(A_{0}+\mu A_{1}\right) h+\sqrt{\mu} B_{1}(y, \gamma) h+\mu B_{2}(y, \gamma, \mu) h=\lambda h \\
\left.h^{\prime}\right|_{y=0}=\left.h^{\prime}\right|_{y=n \pi}=0, \quad '=\frac{d}{d y},
\end{gathered}
$$

где

$$
\begin{gathered}
B_{1}(y, \gamma) h=\frac{\eta_{0}^{2}(\gamma)}{2}[(a, h) D a+D h] \cos ^{2} y \\
B_{2}(y, \gamma, 0) h=\frac{\eta_{0}(\gamma)}{2}\left[(a, h) D w_{1}(y, \gamma)+\left(w_{1}(y, \gamma), h\right) D a+\left(a, w_{1}(y, \gamma)\right) D h\right] \cos y
\end{gathered}
$$

$w_{1}(y, \gamma)$ - результат подстановки $(22)$ в (20) и последующей замены $x$ на $y$, а параметр $\gamma$, принимаюший дискретные значения $\gamma_{n}=\gamma_{n}(\mu)$, для удобства будем считать непрерывно меняюшимся на промежутке (12).

Проблема устойчивости состояния равновесия (14) сводится, очевидно, к анализу расположения спектра задачи (26). В связи с этим напомним, что, как следует из результатов [12], этот спектр состоит из счетного числа собственных значений, а соответствуюшая им система собственных и присоединенных функций образует базис Рисса в пространстве $\stackrel{\circ}{W}_{2}^{2}\left([0, \pi] ; \mathbb{R}^{2}\right)$.

Все возможные пределы при $\mu \rightarrow 0$ собственных значений задачи (26) - это собственные значения матриц

$$
A_{0}-\delta \nu^{2} D, \quad \nu=\frac{k}{n}, \quad k=0,1, \ldots
$$


которые можно получить из уравнения (26) при $\mu=0, h=c \cos y, c \in \mathbb{R}^{2}$. Непосредственная проверка показьвает гурвицевость матрищы (28) при любом $\nu \neq 1$ и существование у нее при всех $\nu$, близких к единице, непрерьвной ветви собственных значений $\lambda(\nu)$ : $\lambda(1)=\lambda^{\prime}(1)=0, \lambda^{\prime \prime}(1)<0$. А это, в свою очередь, означает, что равномерно по $n$ все пределы при $\mu \rightarrow 0$ собственных значений задачи (26) лежат в полуплоскости $\operatorname{Re} \lambda \leqslant 0$.

Итак, исследование устойчивости состояний равновесия (14) сводится к построению асимптотики тех собственных значений задачи (26), которые стремятся к нулю при $\mu \rightarrow 0, n \rightarrow \infty$. В связи с этим заметим, что соответствующие данным собственньгм значениям при $\mu=0$ собственные функции $c \cos (1+z) y, z= \pm k / n, k=0,1, \ldots, c \in \mathbb{R}^{2}$, можно представить в виде

$$
\begin{gathered}
h=h_{1}(y) \cos z y-h_{2}(y) \sin z y \\
\left.\frac{d h_{1}}{d y}\right|_{y=0}=\left.\frac{d h_{1}}{d y}\right|_{y=\pi}=0,\left.\quad h_{2}\right|_{y=0}=\left.h_{2}\right|_{y=\pi}=0 .
\end{gathered}
$$

Поэтому будем искать их в указанном виде и при $\mu>0$.

Подставляя $(29)$ в $(26)$, получим систему $\left({ }^{\prime}=d / d y\right)$

$$
\begin{gathered}
(\delta+\sqrt{\mu} \gamma) D\left(h_{1}^{\prime \prime}-2 z h_{2}^{\prime}-z^{2} h_{1}\right)+\left(A_{0}+\mu A_{1}\right) h_{1} \\
\quad+\sqrt{\mu} B_{1}(y, \gamma) h_{1}+\mu B_{2}(y, \gamma, \mu) h_{1}=\lambda h_{1}, \\
(\delta+\sqrt{\mu} \gamma) D\left(h_{2}^{\prime \prime}+2 z h_{1}^{\prime}-z^{2} h_{2}\right)+\left(A_{0}+\mu A_{1}\right) h_{2} \\
\quad+\sqrt{\mu} B_{1}(y, \gamma) h_{2}+\mu B_{2}(y, \gamma, \mu) h_{2}=\lambda h_{2},
\end{gathered}
$$

которую дополним граничньми условиями (30). Для удобства параметр $z$ (принимаюший дискретные значения $\pm k / n, k=0,1, \ldots$ ) ниже будем считать меняющимся непрерьвно. Из очевидной связи задач $(26)$ и $(31),(30)$ вытекает, что проблема устойчивости состояний равновесия задачи (14) сводится к асимптотическому вычислению собственных значений задачи (31), (30), стремяшихся к нулю при $\mu, z \rightarrow 0$.

При $\mu=z=0$ задача (31), (30) распадается на две независимые краевые задачи $\left({ }^{\prime}=d / d y\right)$

$$
\begin{array}{ll}
\delta D h_{1}^{\prime \prime}+A_{0} h_{1}=\lambda h_{1}, & \left.h_{1}^{\prime}\right|_{y=0}=\left.h_{1}^{\prime}\right|_{y=\pi}=0, \\
\delta D h_{2}^{\prime \prime}+A_{0} h_{2}=\lambda h_{2}, & \left.h_{2}\right|_{y=0}=\left.h_{2}\right|_{y=\pi}=0,
\end{array}
$$

каждая из которых имеет простое нулевое собственное значение с собственньми функциями $h_{1}^{0}(y)=a \cos y$ и $h_{2}^{0}(y)=a \sin y$ соответственно. Остальные собственные значения задач $(32)$ лежат в полуплоскости $\operatorname{Re} \lambda<0$.

Обозначим через $L$ дифференциальньй оператор, порожденный левыми частями уравнений $(31)$ в пространстве $E=\stackrel{\circ}{W}_{2}^{2}\left([0, \pi] ; \mathbb{R}^{4}\right)$ вектор-функций colon $\left(h_{1}, h_{2}\right)$, удовлетворяюших граничным условиям (30). Из изложенного вьше следует, что при всех достаточно малых $\mu$ и $z$ оператор $L$ имеет двумерное инвариантное подпространство

$$
E_{0}(\mu, z)=\operatorname{span}\left\{v_{1}(y, \mu, z), v_{2}(y, \mu, z)\right\} \subset E,
$$

где $v_{j}(y, 0,0)=e_{j}(y), j=1,2, e_{1}(y)=\operatorname{colon}\left(h_{1}^{0}(y), 0\right), e_{2}(y)=\operatorname{colon}\left(0, h_{2}^{0}(y)\right)$. Инвариантность подпространства (33) означает существование матришы второго порядка $\Lambda(\mu, z), \Lambda(0,0)=0$, такой, что

$$
L V=V \Lambda
$$


где по столбцам матрицы $V$ размера $4 \times 2$ стоят базисные функции подпространства $E_{0}(\mu, z)$ (см. (33)). Отметим также, что матрицы $V$ и $\Lambda$ можно выбрать гладко зависяшими от $\sqrt{\mu}$ и $z$, так как от этих параметров гладко зависят коэффициенты уравнений $(31)$.

Итак, проблема устойчивости состояний равновесия (14) свелась к исследованию расположения собственных значений матрищы $\Lambda(\mu, z)$, что, в свою очередь, требует значения нескольких членов ее тейлоровского разложения по $\sqrt{\mu}$ и $z$. Для их нахождения подставим в соотношения (34) разложения

$$
\begin{aligned}
& \Lambda=\mu \Lambda_{1}+\sqrt{\mu} z \Lambda_{2}+z^{2} \Lambda_{3}+\cdots \\
& V=V_{0}+\sqrt{\mu} V_{1}+z V_{2}+\mu V_{3}+\sqrt{\mu} z V_{4}+z^{2} V_{5}+\cdots
\end{aligned}
$$

где

$$
\begin{gathered}
V_{0}=\left[e_{1}(y), e_{2}(y)\right], \quad V_{j}=\left[v_{1 j}, v_{2 j}\right], \quad j=1, \ldots, 5, \\
v_{1 j}=\operatorname{colon}\left(h_{1 j}, 0\right), \quad v_{2 j}=\operatorname{colon}\left(0, h_{2 j}\right), \quad j=1,3,5, \\
v_{1 j}=\operatorname{colon}\left(0, h_{1 j}\right), \quad v_{2 j}=\operatorname{colon}\left(h_{2 j}, 0\right), \quad j=2,4, \\
\Lambda_{1}=\left(\begin{array}{cc}
\lambda_{1} & 0 \\
0 & \lambda_{2}
\end{array}\right), \quad \Lambda_{2}=\left(\begin{array}{cc}
0 & \lambda_{3} \\
\lambda_{4} & 0
\end{array}\right), \quad \Lambda_{3}=\left(\begin{array}{cc}
\lambda_{5} & 0 \\
0 & \lambda_{6}
\end{array}\right) .
\end{gathered}
$$

Приравнивая затем коэффициенты при одинаковьх степенях $\sqrt{\mu}$ и $z$, для определения функций $v_{1 j}, v_{2 j}, j=1, \ldots, 5$, получим рекуррентную последовательность краевых задач, из условий разрешимости которых находим элементы матриц $\Lambda_{j}, j=1,2,3$.

Действуя указанным вьше способом, для $h_{1 j}, h_{2 j}, j=1,2$, получим краевые задачи $\left(L_{0}=\delta D d^{2} / d y^{2}+A_{0}\right):$

$$
\begin{array}{rlrl}
L_{0} h_{11}+\left(B_{1}(y, \gamma)-\gamma D\right) a \cos y & =0, & \left.\frac{d h_{11}}{d y}\right|_{y=0}=\left.\frac{d h_{11}}{d y}\right|_{y=\pi}=0 \\
L_{0} h_{21}+\left(B_{1}(y, \gamma)-\gamma D\right) a \sin y & =0, & & \left.h_{21}\right|_{y=0}=\left.h_{21}\right|_{y=\pi}=0 \\
L_{0} h_{12}-2 \delta D a \sin y & =0, & & \left.h_{12}\right|_{y=0}=\left.h_{12}\right|_{y=\pi}=0 \\
L_{0} h_{22}-2 \delta D a \cos y & =0, & \left.\frac{d h_{22}}{d y}\right|_{y=0}=\left.\frac{d h_{22}}{d y}\right|_{y=\pi}=0
\end{array}
$$

разрешимые по той же причине, что и задача (18). Учитывая здесь явньй вид матрицы $B_{1}(y, \gamma)($ см. $(27))$, находим (векторы $a, a_{1}$ те же, что и в $\left.(20)\right)$ :

$$
\begin{array}{ll}
h_{11}=a_{1}\left(\gamma-\frac{9}{8} \eta_{0}^{2}\right) \cos y+\frac{3 \eta_{0}^{2}}{64 \delta} a \cos 3 y, & h_{12}=2 \delta a_{1} \sin y, \\
h_{21}=a_{1}\left(\gamma-\frac{3}{8} \eta_{0}^{2}\right) \sin y+\frac{3 \eta_{0}^{2}}{64 \delta} a \sin 3 y, & h_{22}=2 \delta a_{1} \cos y .
\end{array}
$$

Приравнивая в (34) коэффициенты при $\mu, \sqrt{\mu} z, z^{2}$ для $h_{1 j}, h_{2 j}, j=3,4,5$, приходим к шести краевым задачам, из которых вьпишем для примера только две $\left({ }^{\prime}=d / d y\right)$ :

$$
\begin{aligned}
& L_{0} h_{13}+\gamma D h_{11}^{\prime}+A_{1} a \cos y+B_{1}(y, \gamma) h_{11}+B_{2}(y, \gamma, 0) a \cos y=\lambda_{1} a \cos y, \\
& \left.\quad h_{13}^{\prime}\right|_{y=0}=\left.h_{13}^{\prime}\right|_{y=\pi}=0 ; \\
& L_{0} h_{23}+\gamma D h_{21}^{\prime \prime}+A_{1} a \sin y+B_{1}(y, \gamma) h_{21}+B_{2}(y, \gamma, 0) a \sin y=\lambda_{2} a \sin y \\
& \left.\quad h_{23}\right|_{y=0}=\left.h_{23}\right|_{y=\pi}=0 .
\end{aligned}
$$


Подставляя во все шесть краевых задач известные функции (35) и учитывая (27), из условий разрешимости этих задач определяем

$$
\lambda_{1}=-4(\gamma+1), \quad \lambda_{2}=0, \quad \lambda_{3}=4 \delta, \quad \lambda_{4}=\frac{4 \delta}{3}(2 \gamma+5), \quad \lambda_{5}=\lambda_{6}=-4 \delta^{2} .
$$

Полученной информации о матрице $\Lambda(\mu, z)$ достаточно для исследования знаков ее определителя и следа при всех $\mu,|z| \ll 1$. Действительно, функции $\operatorname{tr} \Lambda(\mu, z)$ и $\operatorname{det} \Lambda(\mu, z)$ четные по $z$ и $\operatorname{det} \Lambda(\mu, 0) \equiv 0$, так как, во-первых, задача $(31),(30)$ не изменяется при заменах $z \rightarrow-z, h_{2} \rightarrow-h_{2}$, а, во-вторых, при $z=0$ она имеет нулевое собственное значение с собственной функцией

$$
\operatorname{colon}\left(0, \frac{\partial w_{0}}{\partial y}(y, \gamma, \mu)\right)
$$

где $w_{0}$ - функция, фигурируюшая в (14). Отсюда и из (36) заключаем, что

$$
\begin{aligned}
\operatorname{tr} \Lambda & =-4(\gamma+1) \mu-8 \delta^{2} z^{2}+O\left(\mu^{3 / 2}+\sqrt{\mu} z^{2}+z^{4}\right), \\
\operatorname{det} \Lambda & =16 \delta^{2} z^{2}\left[\frac{1}{3}(\gamma-2) \mu+\delta^{2} z^{2}+O\left(\mu^{3 / 2}+\sqrt{\mu} z^{2}+z^{4}\right)\right] .
\end{aligned}
$$

Из (38) вытекает отрицательность $\operatorname{tr} \Lambda$ при любом фиксированном $\gamma>-1$ и при всех достаточно малых $\mu>0,|z|$. Далее, если для отрезка (12) потребовать выполнение условия

$$
\left[\gamma_{1}, \gamma_{2}\right] \subset(2, \infty)
$$

то, как следует из $(39)$, при всех $0<\mu \ll 1,0<|z| \ll 1$ будет вьполняться и неравенство $\operatorname{det} \Lambda>0$.

Проделанньй анализ показьвает, что критические собственные числа задачи (26) являются собственньми значениями матриц

$$
\Lambda_{k}=\Lambda\left(\mu, \frac{k}{n}\right), \quad k=0, \pm 1, \ldots, \pm k_{0},
$$

где $k_{0}$ - целая часть $n z_{0}$, а $z_{0}>0$ фиксировано и достаточно мало. Заметим, что при условии (40) все они являются гурвищевыми. Исключение составляет только матрица (41) с номером $k=0$, одно собственное значение которой отрицательно, а другое равно нулю. Однако при переходе от $(31),(30)$ к (26) нулевое собственное число пропадает, ибо в силу (29), (37) ему отвечает нулевая собственная функция.

Суммируя изложенное, приходим к следующему утверждению.

Лемма 2. При условии (40) все состояния равновесия (14), (15) краевой задачи (6), доставляемые леммой 1 и принципом подобия, әкспоненциально устойчиsbl.

Из приведенного вьше обоснования леммы 2 следует, что ее утверждение сохраняется и в случае, когда $\beta$ является независимым от $\mu$ мальм параметром. Заметим, далее, что для любого фиксированного отрезка вида (40) количество $n_{*}$ номеров $n$, удовлетворяющих неравенствам (15), при $\beta \rightarrow 0$ равномерно по $\mu$ имеет порядок $\beta^{-1}$. Таким образом, при $\beta \rightarrow 0$ и при фиксированных прочих параметрах количество устойчивых состояний равновесия задачи (6) неограниченно растет. Добавим еше, что поскольку 
$n_{*} \sim\left(\gamma_{2}-\gamma_{1}\right) /(2 \sqrt{\delta} \beta)$ при $\mu \rightarrow 0$, то неограниченного увеличения числа устойчивых состояний равновесия (14) можно добиться и другим способом, а именно, за счет увеличения длины отрезка (40) и надлежащего уменьшения $\mu$ (при фиксированном $\beta$ ). Теорема 2 доказана.

В заключение отметим, что изучение явления буферности в гиперболических уравнениях берет начало с заметки [13], в которой исследовалось нелинейное телеграфное уравнение с малой диффузией. В дальнейшем это явление удалось обнаружить у широкого класса уравнений волнового типа как в сингулярно возмущенной постановке, так и в гладком случае (см. монографию [14], где соответствующие результаты изложены в суммированном виде).

\section{СПИСОК ЦИТИРОВАННОЙ ЛИТЕРАТУРЫ}

[1] Боголюбов Н. Н., Митропольский Ю. А. Асимптотические методы в теории нелинейных колебаний. М.: Наука, 1974.

[2] Митропольский Ю. А., Мосеенков Б. И. Асимптотические решения уравнений в частных производных. Киев: Вища школа, 1976.

[3] Колесов А. Ю., Колесов Ю. С. Принцип сведения Боголюбова-Митропольского в задаче о параметрическом возбуждении автоволн // Докл. АН СССР. 1989. Т. 307. № 4. C. $837-840$.

[4] Колесов Ю. С. Нелинейный параметрический резонанс в сингулярно возмущенном телеграфном уравнении // Дифференц. уравнения. 1991. Т. 27. №10. С. 1828-1829.

[5] Колесов А. Ю. Параметрические колебания решений телеграфного уравнения с умеренно малой диффузией // Сиб. матем. ж. 1992. Т. 33. №6. С. 79-86.

[6] Колесов Ю.С. Асимптотика и устойчивость нелинейных параметрических колебаний сингулярно возмущенного телеграффного уравнения // Матем. сб. 1995. Т. 186. № 10. С. 57-72.

[7] Колесов Ю. С. Параметрические колебания сингулярно возмущенного телеграфного уравнения с маятниковой нелинейностью // Матем. сб. 1998. Т. 189. № 3. С. 69-82.

[8] Колесов А. Ю., Мищенко Е.Ф., Розов Н. Х. Явление параметрической буферности в системах параболических и гиперболических уравнений с малой диффузией // Укр. матем. ж. 1998. Т. 50. № 1. С. 22-35.

[9] Колесов А. Ю., Розов Н. Х. О существовании асимптотически большого числа устойчивых диссипативных структур в параболических системах с малой диффузией // Тр. семинара им. И. Г. Петровского. 1997. Т. 20. С. 3-26.

[10] Гленсдорф П., Пригожин И. Термодинамическая теория структуры, устойчивости и флюктуаций. М.: Мир, 1973.

[11] Митропольский Ю.А., Лыкова О. Б. Интегральные многообразия в нелинейной механике. М.: Наука, 1973.

[12] Наймарк М. А. Линейные дифференциальные операторы. М.: Наука, 1969.

[13] Колесов А. Ю., Колесов Ю. С. Бифуркация автоколебаний сингулярно возмущенного волнового уравнения // Докл. АН СССР. 1990. Т. 315. № 2. С. 281-283.

[14] Колесов А. Ю., Мищенко Е. Ф., Розов Н. Х. Асимптотические методы исследования периодических решений нелинейных гиперболических уравнений // Тр. МИАН. 1998. Т. 222.

(А. Ю. Колесов) Ярославский государственный университет им. П.Г. Демидова

Поступило

(Н. Х. Розов) Московский государственный университет им. М.В. Ломоносова

31.01.2000 